\title{
The Safety and Efficacy of Treatment with Nab-paclitaxel and Carboplatin for Patients with Advanced Squamous Non-small Cell Lung Cancer Concurrent with Idiopathic Interstitial Pneumonias
}

\author{
Tetsuo Fujita ${ }^{1}$, Takuma Hiroishi ${ }^{1}$, Kohei Shikano ${ }^{1}$, Asako Yanagisawa ${ }^{1}$, Noriko Hayama ${ }^{1}$, \\ Hiroyuki Amano ${ }^{1}$, Makoto Nakamura ${ }^{1}$, Satoshi Hirano ${ }^{2}$, \\ Hiroshi Tabeta ${ }^{1}$ and Sukeyuki Nakamura ${ }^{1}$
}

\begin{abstract}
:
Objective Although lung squamous cell carcinoma (SCC) accounts for $20-30 \%$ of lung cancer cases, new treatment options are limited. The CA031 study showed that nanoparticle albumin-bound-paclitaxel (nabPTX) plus carboplatin produced a significantly higher overall response rate $(41 \%)$ than solvent-based paclitaxel plus carboplatin in patients with lung SCC. However, the safety and efficacy of combination chemotherapy of nab-PTX and carboplatin has not yet been established for patients with concurrent lung SCC and idiopathic interstitial pneumonias (IIPs). The aim of this study was to assess the safety and efficacy profiles of nab-PTX and carboplatin in patients with lung SCC and concurrent IIPs.

Methods Eight patients with inoperable-stage lung SCC and IIPs were treated with nab-PTX plus carboplatin in a first-line setting between June 2013 and December 2016. One of the eight was a woman, and the median age was 77 (range $=72-80$ ) years. Their clinical outcomes, including chemotherapy-associated acute exacerbation of IIPs, were retrospectively investigated.

Results The overall response rate was 50\%, the median progression-free survival time was 5.6 months, and the median overall survival time was 8.1 months. No patients experienced chemotherapy-related exacerbation of IIPs in the first-line treatment with nab-PTX plus carboplatin. However, IIPs worsened in two of four patients who received second-line chemotherapy.

Conclusion Combination chemotherapy of nab-PTX and carboplatin may be an effective and safe treatment option for patients with inoperable lung SCC with IIPs. To confirm this, a large-scale prospective study is needed.
\end{abstract}

Key words: squamous cell carcinoma, idiopathic interstitial pneumonias, nanoparticle albumin-boundpaclitaxel, carboplatin, chemotherapy

(Intern Med 57: 1827-1832, 2018)

(DOI: 10.2169/internalmedicine.0404-17)

\section{Introduction}

Lung cancer is the leading cause of cancer mortality worldwide, and non-small cell lung cancer (NSCLC) accounts for more than $80 \%$ of all lung cancer cases.
Platinum-based doublet chemotherapy is considered a standard regimen for patients with advanced NSCLC. Solventbased paclitaxel plus carboplatin (sb-PC) has been the most commonly used taxane-platinum combination in the United States and Japan and is associated with an overall response rate (ORR) of $17-32 \%$ and a median overall survival (OS)

${ }^{1}$ Department of Respirology, Funabashi Municipal Medical Center, Japan and ${ }^{2}$ Department of Medical Oncology, Funabashi Municipal Medical Center, Japan

Received: October 24, 2017; Accepted: November 13, 2017; Advance Publication by J-STAGE: February 9, 2018

Correspondence to Dr. Tetsuo Fujita, phajita0318@yahoo.co.jp 
Table 1. Patient Characteristics $(n=8)$.

\begin{tabular}{ll}
\hline \multicolumn{1}{c}{ Characteristics } & \multicolumn{1}{c}{ Value } \\
\hline Gender (male/female) & $7 / 1$ \\
Median age (range) (years) & $77(72-80)$ \\
Smoking status (never/former/current) & $0 / 4 / 4$ \\
Performance status (0/1/2/3) & $3 / 5 / 0 / 0$ \\
Stage (IIIa/IIIb/IV) & $5 / 1 / 2$ \\
Combined with emphysema (yes/no) & $5 / 3$ \\
IIPs radiological pattern (UIP/non-UIP) & $4 / 4$ \\
Median \%VC (predicted) (\%) (range) & 81.5 (69.6-115.8) \\
\hline IIPs: idiopathic interstitial pneumonias, UIP: usual interstitial pneu- \\
monia, \%VC: \%vital capacity
\end{tabular}

of 8.1-12.3 months $(1,2)$. Recently, treatment with nanoparticle albumin-bound-paclitaxel (nab-PTX) plus carboplatin (nab-PC) was approved for chemotherapy-naïve advanced NSCLC. This approval was based on the results of the CA031 study, in which nab-PC demonstrated a higher ORR than sb-PC (33\% vs. $25 \%, \mathrm{p}=0.005)$ (3). In addition, in a subgroup analysis of the CA031 study, there was a significant improvement in the ORR of patients with lung squamous cell carcinoma (SCC) $(41 \%$ vs. $24 \%, \mathrm{p}<0.001)$ without a significant increase in the OS (4).

Interstitial lung disease (ILD) is correlated with a high incidence of lung cancer $(5,6)$, and preexisting ILD is considered a risk factor for chemotherapy-related ILD (7-9). Therefore, patients with NSCLC and preexisting ILD are at risk of chemotherapy-related acute exacerbation of ILD (AE-ILD), which is a potentially fatal complication of lung cancer treatment (9). Although standard regimens for NSCLC patients with preexisting ILD have not yet been established, among platinum-based doublet regimens available for ILD, sb-PC has been the most frequently used and evaluated regimen for first-line chemotherapy in patients with advanced NSCLC and ILD (10). A previous study showed the incidence of chemotherapy-related AE-ILD was $5.6 \%$ in NCSLC patients with ILD who received sb-PC as first-line therapy (11). Considering the prevalence of AEILD in patients with idiopathic pulmonary fibrosis or idiopathic nonspecific interstitial pneumonia even without lung cancer $(8.5 \%$ or $4.2 \%$ in a 1-year period, respectively) $(12,13)$, the incidence of AE-ILD by chemotherapy with sb-PC regimen might be within the permissible range. Furthermore, recent reports have revealed an addition of bevacizumab to sb-PC might provide an effective and safe treatment option for patients with advanced non-squamous NSCLC and ILD (14-16). However, the optimum chemotherapy regimen for advanced lung SCC with preexisting ILD has not been determined.

Given the above, we investigated the safety and efficacy of nab-PC for patients with advanced lung SCC with ILD.

\section{Materials and Methods}

\section{Patients and treatment methods}

We retrospectively examined eight patients of unresectable stage lung SCC with preexisting ILD who received nab-PC in a first line setting at Funabashi Municipal Medical Center between Jun 2013 and December 2016. Carboplatin was administered at a dose of area under the curve (AUC) $5-6 \mathrm{mg} /$ $\mathrm{mL} / \mathrm{min}$ on day 1 and nab-PTX at a dose of $100 \mathrm{mg} / \mathrm{m}^{2}$ was administered on days 1,8 and 15 every 4 weeks. Each fourweek treatment schedule was designated as a single cycle. ILD was diagnosed based on clinical features and computed tomography (CT) images obtained prior to chemotherapy. The presence of ILD was evaluated by at least two pulmonologists in accordance with the clinical criteria established by the American Thoracic Society (ATS)/European Respiratory Society (ERS) (17).

\section{Adverse events}

Adverse events were evaluated using the Common Terminology Criteria for Adverse Events (CTCAE) version 4.0. Chemotherapy-related AE-ILD was diagnosed based on the CT findings as follows: new bilateral ground-glass abnormality and/or consolidation superimposed on a background of interstitial shadow with the exclusion of other diseases, such as heart failure, pulmonary infection and pulmonary embolism $(18,19)$.

\section{Clinical evaluations and statistical analyses}

CT was performed within one month before initiating treatment to assess the primary tumor and repeated every two to three months. All responses were defined according to Response Evaluation Criteria in Solid Tumor (RECIST). The progression-free survival (PFS) was measured from the start of treatment to the time of progression. The overall survival (OS) was measured from the start of treatment until death. The event time was analyzed using the Kaplan-Meier method.

\section{Results}

\section{Patient characteristics}

The clinical characteristics are summarized in Table 1. Only one of the eight patients was a woman, and the median age was 77 years (range, 72-80 years). All patients were current or previous smokers, and five also had pulmonary emphysema. All patients had an Eastern Cooperative Oncology Group (ECOG) performance status of 0 or 1 . Six patients had stage III and two had stage IV disease. There were no patents with collagen vascular disease or a history of exposure to dust or asbestos. Therefore, ILD in all patients was diagnosed as idiopathic interstitial pneumonia (IIP). The IIP pattern was usual interstitial pneumonia (UIP) 
Table 2. Treatment Response.

\begin{tabular}{lc}
\hline \multicolumn{1}{c}{ Response } & $\mathrm{n}$ \\
\hline Complete response & 0 \\
Partial response & 4 \\
Stable disease & 3 \\
Progressive disease & 1 \\
\hline & $\%$ \\
\hline Overall response rate & 50.0 \\
Disease control rate & 87.5 \\
1-year survival rate & 37.5 \\
\hline
\end{tabular}

Table 3. Treatment-related Adverse Events.

\begin{tabular}{lccccc}
\hline \multirow{2}{*}{ Toxicity } & \multicolumn{5}{c}{ Grade } \\
\cline { 2 - 6 } & 1 & 2 & 3 & 4 & $3-5(\%)$ \\
\hline Neutropenia & 1 & 1 & 5 & 0 & 62.5 \\
Thrombocytopenia & 6 & 0 & 0 & 0 & 0 \\
Anemia & 3 & 5 & 0 & 0 & 0 \\
Febrile neutropenia & - & - & 0 & 0 & 0 \\
Peripheral neuropathy & 2 & 2 & 0 & 0 & 0 \\
Anorexia & 2 & 2 & 0 & 0 & 0 \\
Nausea & 0 & 1 & 0 & 0 & 0 \\
Fatigue & 0 & 1 & 0 & 0 & 0 \\
\hline
\end{tabular}

in four patients. The median \%vital capacity (\%VC: VC/predicted VC) was $81.5 \%$ (range 69.6-115.8\%).

\section{Treatment exposure}

The median number of treatment cycles was 4 (range 2-5 cycles). Carboplatin was administered at a dose of AUC 6 in one patient and AUC 5 in the others, and in five patients, nab-PTX was not administered on day 15 for one whole cycle. The median cumulative nab-PTX dose was $850 \mathrm{mg} / \mathrm{m}^{2}$, with a median nab-PTX intensity of $62 \mathrm{mg} / \mathrm{m}^{2} /$ week. The median cumulative carboplatin dose was $1,712 \mathrm{mg}$, with a median carboplatin intensity of $71 \mathrm{mg} / \mathrm{m}^{2} /$ week. Half of these patients received second-line chemotherapy.

\section{Efficacy}

The overall response rate was $50 \%$, and the disease control rate was $87.5 \%$ (Table 2). The 1-year survival rate was $37.5 \%$. The median PFS was 5.6 months (Figure a), and the median OS was 8.1 months (Figure b). In the second-line setting, the overall response rate was $0 \%$.

\section{Toxicity}

The toxicities of the treatments with nab-PC are summarized in Table 3. Regarding hematological adverse events, 5 $(62.5 \%)$ patients developed grade 3 neutropenia, but no patients exhibited $\geq$ grade 3 anemia, leukocytopenia or thrombocytopenia. In addition, no patients experienced febrile neutropenia or $\geq$ grade 3 non-hematological adverse events. The most frequent non-hematological adverse events were peripheral neuropathy and anorexia in 4 patients $(50 \%)$. The
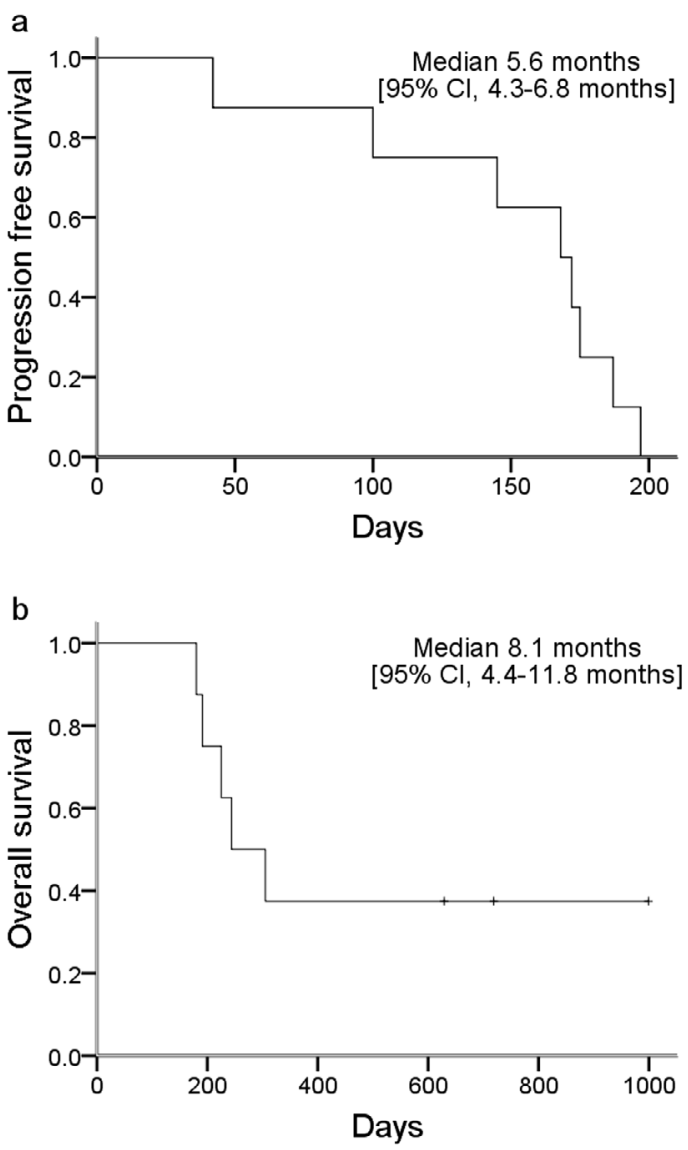

Figure. Kaplan-Meier curves for the progression-free survival (a) and overall survival (b).

planned day 15 dose was omitted in 7 patients (88\%) because of grade 3 neutropenia or at the discretion of the attending physician. There was one instance of discontinuation of treatment at the patient's discretion. No patients experienced chemotherapy-related exacerbation of IIPs during the first-line treatment with nab-PC. However over the whole period, acute exacerbation of IIPs (AE-IIPs) developed in two patients who received second-line therapy. They were treated with docetaxel monotherapy and vinorelbin in combination with carboplatin and concurrent electron beam therapy for chest wall invasion. Both patients (Stage IIIa/nonUIP pattern and Stage IIIb/UIP pattern) died due to AE-IIPs with a diffuse alveolar damage pattern and survived for 8.1 and 6.0 months, respectively.

\section{Discussion}

To our knowledge, this is the first study to evaluate the safety and efficacy of nab-PC as a first-line treatment for patients with advanced lung SCC with IIPs. The CA031 study showed that nab-PC has a favorable risk-benefit profile compared to sb-PC (3). In the subset analysis for advanced lung SCC, nab-PC produced a significantly higher ORR (41\% vs. $24 \%$ ) and a more than 1-month increase in the OS over sb-PC (median 10.7 vs. 9.5 months) without an improvement in the PFS (median 5.6 months vs. 5.7 
Table 4. Comparison of Dose Delivery in the CA031 Study and the Present Study.

\begin{tabular}{|c|c|c|c|c|c|}
\hline \multirow[b]{2}{*}{ Subgroup } & \multirow[b]{2}{*}{ Cycles } & \multicolumn{2}{|c|}{ nab-paclitaxel } & \multicolumn{2}{|c|}{ carboplatin } \\
\hline & & $\begin{array}{l}\text { Cumulative dose } \\
\qquad\left(\mathrm{mg} / \mathrm{m}^{2}\right)\end{array}$ & $\begin{array}{l}\text { Dose intensity } \\
\left(\mathrm{mg} / \mathrm{m}^{2} / \text { week }\right)\end{array}$ & $\begin{array}{l}\text { Cumulative dose } \\
\left(\mathrm{mg} / \mathrm{m}^{2}\right)\end{array}$ & $\begin{array}{l}\text { Dose intensity } \\
\left(\mathrm{mg} / \mathrm{m}^{2} / \text { week }\right)\end{array}$ \\
\hline \multicolumn{6}{|c|}{ Patients without preexisting ILD (The CA031 study) } \\
\hline NSCLC [3] & 6 & 1,325 & 82 & 3,140 & 166 \\
\hline Lung SCC [4] & 6 & 1,475 & 85.2 & N/A & N/A \\
\hline Elderly NSCLC [20] & 5 & 1,150 & 73.4 & 2,428 & 127.7 \\
\hline \multicolumn{6}{|c|}{ Patients with preexisting ILD (The present study) } \\
\hline Elderly lung SCC & 4 & 850 & 62 & 1,712 & 71 \\
\hline
\end{tabular}

nab-paclitaxel: nanoparticle albumin-bound-paclitaxel, ILD: interstitial lung disease, NSCLC: non-small cell lung cancer, SCC: squamous cell carcinoma, N/A: not available

Table 5. Comparison of Treatment Efficacy in the CA031 Study and the Present Study.

\begin{tabular}{|c|c|c|c|c|c|c|}
\hline Subgroup & $\mathrm{n}$ & mAge (y) & ORR $(\%)$ & mPFS (m) & mOS (m) & 2nd line $(\%)$ \\
\hline \multicolumn{7}{|c|}{ Patients without preexisting ILD (The CA031 study) } \\
\hline NSCLC [3] & 521 & 60 & 33 & 6.3 & 12.1 & 53 \\
\hline Lung SCC [4] & 229 & 59 & 41 & 5.6 & 10.7 & 43 \\
\hline Elderly NSCLC [20] & 74 & 72 & 34 & 8 & 19.9 & 61 \\
\hline \multicolumn{7}{|c|}{ Patients with preexisting ILD (The present study) } \\
\hline Elderly lung SCC & 8 & 77 & 50 & 5.6 & 8.1 & 50 \\
\hline
\end{tabular}

m: median, (y): years old, ORR: overall response rate, PFS: progression-free survival, (m): months, OS: overall survival, 2nd line: the proportion of patients treated with second-line therapy, ILD: interstitial lung disease, NSCLC: non-small cell lung cancer, SCC: squamous cell carcinoma, N/A: not available

months) (4). The ORR of $50 \%$ and PFS of 5.6 months in the present study are consistent with those in the CA031 study. With regard to the treatment methods used in the CA031 study, nab-PTX at a dose of $100 \mathrm{mg} / \mathrm{m}^{2}$ was administered on days 1,8 , and 15 every 3 weeks followed by carboplatin at a dose of AUC 6 on day 1, and the median number of cycles was 6 (range 1-31 cycles) (3). Therefore the median cumulative doses and intensities of nab-PTX $(1,325$ $\mathrm{mg} / \mathrm{m}^{2}$ and $82 \mathrm{mg} / \mathrm{m}^{2} /$ week) and carboplatin $(3,140 \mathrm{mg}$ and $166 \mathrm{mg} / \mathrm{m}^{2} /$ week) used in the CA 031 study were markedly higher than those used in the present study (Table 4).

All patients enrolled in the present study were elderly Japanese patients ( $\geq 70$ years of age). A subgroup analysis of the CA031 study also showed the safety and efficacy of nab-PC in elderly patients ( $\geq 70$ years of age), resulting in an ORR of $34 \%$, PFS of 8.0 months and OS of 19.9 months (20). The median dose intensities of nab-PTX and carboplatin used in the elderly subgroup were $73.4 \mathrm{mg} / \mathrm{m}^{2} /$ week and $127.7 \mathrm{mg} / \mathrm{m}^{2} /$ week, respectively, which are also higher than those used in the present study (Table 4). Recently, several phase II studies suggested that dose reduction in the nab-PC regimen might be required for elderly Japanese patients $(21,22)$. The dose intensities of nab-PTX and carboplatin used in those studies were $45-50 \mathrm{mg} / \mathrm{m}^{2} /$ week and $118.5 \mathrm{mg} /$ week, respectively, which were lower than those used in the elderly subgroup of the CA031 study. However, the low dose of the nab-PC regimen produced sig- nificant efficacy with acceptable toxicities, resulting in an ORR of 50\%, PFS of 6.4-7.1 months and OS of 17.5-19.1 months, although the sample size was small. These findings may be consistent with the results of the present study, in which lower cumulative doses and intensities of both drugs did not lead to a poor ORR or short PFS in Japanese elderly patients with lung SCC and IIPs.

The present study showed a poor survival compared to the subgroup analyses of the CA031 study (Table 5) $(3,4,20)$. Considering the relatively little difference in the median PFS and proportion of patients treated with second-line therapy between the present study and the CA031 study, the poor survival in the present study may be due to the high incidence of fatal AE-IIPs (in two of four patients) related to second-line chemotherapy. One of these two patients received docetaxel monotherapy, and the other was treated with cisplatin and vinorelbine in the second-line setting. The latter patient had chest pain due to tumor invasion into the chest wall and needed palliative electron therapy, which is commonly used in the treatment of superficial tumors. Regarding the role of second-line chemotherapy in patients with NSCLC and preexisting ILD, previous reports described a higher risk of developing AE-ILD and lower treatment effect with second-line docetaxel monotherapy than with first-line platinum-based chemotherapy $(23,24)$. Okuda et al. reported that combination therapy with vinorelbine plus a platinum agent was a suitable treatment option 
for patients with advanced NSCLC and preexisting ILD, although the ratio of AE-ILD was $15.8 \%$ (3/19 patients), including 1 fatal case (25). However, their study targeted chemotherapy-naïve patients. The safety and efficacy of second-line chemotherapy with vinorelbine plus a platinum agent for patients with NSCLC and ILD has never been evaluated. Furthermore, doublet chemotherapy as a secondline treatment is more toxic and does not improve the OS compared to single-agent treatment (26). Kenmotsu et al. showed that chemotherapy-related AE-ILD occurred in 30\% of patients who received second-line or subsequent chemotherapy after platinum-based chemotherapy. The development of AE-ILD related to second-line or subsequent chemotherapy may shorten the lifespan and lead to a poor survival in patients with advanced NSCLC. These findings suggest that the risks and benefits must be carefully evaluated before administering second-line chemotherapy to patients with ILD.

Recently, other groups have also reported the favorable efficacy and safety of the nab-PC regimen for treating advanced NSCLC patients with $\operatorname{ILD}(27,28)$. Reported values for the median ORR, PFS and OS were 56-67\%, 5.1-5.8 months and 11.5-14.9 months, respectively, and AE-ILD developed in $0-8.3 \%$. Regarding the sb-PC regimen in patients with NSCLC and preexisting ILD, several previous studies showed that the median ORR, PFS and OS were 27-61\%, 2.5-5.3 months and 7.0-10.6 months, respectively, and AEILD developed in $0-26.7 \%(10,11,15,29)$. Therefore, the efficacy and safety of the nab-PC regimen seem to be comparable to those of the sb-PC regimen. However, these studies focused on NSCLC or non-squamous NSCLC, and whether or not the nab-PC regimen is superior to the sb-PC regimen in patients with lung SCC and preexisting ILD remains unclear.

A new treatment with immune checkpoint inhibitors such as nivolumab and pembrolizumab [programmed death 1 (PD-1) inhibitors] has shown benefits in patients with lung SCC, but the incidence of drug-induced ILD was reported to be higher in patients treated with PD-1 inhibitors than in those treated with cytotoxic agents in 2 open-label phase 3 trials $(5 \%$ in the nivolumab group vs. $0 \%$ in the docetaxel group, $5.8 \%$ in the pembrolizumab group vs. $0.7 \%$ in the platinum-based chemotherapy group) $(30,31)$. In contrast, the CA031 study did not report any chemotherapy-induced ILD (3). These findings indicate that chemotherapy with a nab-PC regimen may be a reasonable cancer therapy for patients with preexisting ILD compared with immune checkpoint inhibitors.

Several limitations associated with the present study warrant mention. First, the study was a retrospective analysis and was performed at a single institution. Second, the diagnosis of preexisting ILD and AE-IIPs was based on the CT findings and not on histological examinations. Third, the patient population was very small, and all patients were Japanese. Finally, the study included five patients with clinical stage IIIa disease. Patients with locally advanced unre- sectable disease may achieve a long-term survival with radiation therapy combined with chemotherapy. However, several studies showed that the presence of preexisting ILD was a significant risk for severe radiation pneumonitis and treatment-related death after radiotherapy and concurrent chemoradiotherapy (32-36). Further studies are required to determine the best treatment option for clinical stage IIIa disease in NSCLC patients with preexisting ILD.

Treatments for advanced NCSLC have been diversified with an improved understanding of the molecular pathways that drive malignancy in NSCLC. Even in patients with NSCLC and preexisting ILD, we may be approaching an era in which personalized medicine is required to achieve the best outcome. The present findings suggest that treatment with nab-PC may be an effective and feasible regimen for patients with lung SCC and IIPs. Further assessment in a large prospective study is needed to confirm these findings.

The authors state that they have no Conflict of Interest (COI).

\section{Acknowledgement}

We thank Chihiro George Kurokawa of the United States for editing our manuscript.

\section{References}

1. Schiller JH, Harrington D, Belani CP, et al. Comparison of four chemotherapy regimens for advanced non-small-cell lung cancer. N Engl J Med 346: 92-98, 2002.

2. Ohe Y, Ohashi Y, Kubota K, et al. Randomized phase III study of cisplatin plus irinotecan versus carboplatin plus paclitaxel, cisplatin plus gemcitabine, and cisplatin plus vinorelbine for advanced non-small-cell lung cancer: four-arm cooperative study in Japan. Ann Oncol 18: 317-323, 2007.

3. Socinski MA, Bondarenko I, Karaseva NA, et al. Weekly nabpaclitaxel in combination with carboplatin versus solvent-based paclitaxel plus carboplatin as first-line therapy in patients with advanced non-small-cell lung cancer: final results of a phase III trial. J Clin Oncol 30: 2055-2062, 2012.

4. Socinski MA, Okamoto I, Hon JK, et al. Safety and efficacy analysis by histology of weekly nab-paclitaxel in combination with carboplatin as first-line therapy in patients with advanced non-small-cell lung cancer. Ann Oncol 24: 2390-2396, 2013.

5. Raghu G, Nyberg F, Morgan G. The epidemiology of interstitial lung disease and its association with lung cancer. Br J Cancer 91 Suppl 2: S3-S10, 2004.

6. Park J, Kim DS, Shim TS, et al. Lung cancer in patients with idiopathic pulmonary fibrosis. Eur Respir J 17: 1216-1219, 2001.

7. Kenmotsu H, Naito T, Kimura M, et al. The risk of cytotoxic chemotherapy-related exacerbation of interstitial lung disease with lung cancer. J Thorac Oncol 6: 1242-1246, 2011.

8. Isobe K, Hata Y, Sakamoto S, Takai Y, Shibuya K, Homma S. Clinical characteristics of acute respiratory deterioration in pulmonary fibrosis associated with lung cancer following anti-cancer therapy. Respirology 15: 88-92, 2010.

9. Kudoh S, Kato H, Nishiwaki Y, et al. Interstitial lung disease in Japanese patients with lung cancer: a cohort and nested casecontrol study. Am J Respir Crit Care Med 177: 1348-1357, 2008.

10. Kenmotsu H, Naito T, Mori K, et al. Effect of platinum-based chemotherapy for non-small cell lung cancer patients with interstitial lung disease. Cancer Chemother Pharmacol 75: 521-526, 2015.

11. Minegishi Y, Sudoh J, Kuribayasi H, et al. The safety and efficacy 
of weekly paclitaxel in combination with carboplatin for advanced non-small cell lung cancer with idiopathic interstitial pneumonias. Lung Cancer 71: 70-74, 2011.

12. Kim DS, Park JH, Park BK, Lee JS, Nicholson AG, Colby T. Acute exacerbation of idiopathic pulmonary fibrosis: frequency and clinical features. Eur Respir J 27: 143-150, 2006.

13. Park IN, Kim DS, Shim TS, et al. Acute exacerbation of interstitial pneumonia other than idiopathic pulmonary fibrosis. Chest 132: 214-220, 2007.

14. Suzuki H, Hirashima T, Kobayashi M, et al. Carboplatin plus paclitaxel in combination with bevacizumab for the treatment of adenocarcinoma with interstitial lung diseases. Mol Clin Oncol 1: 480-482, 2013.

15. Shimizu R, Fujimoto D, Kato R, et al. The safety and efficacy of paclitaxel and carboplatin with or without bevacizumab for treating patients with advanced nonsquamous non-small cell lung cancer with interstitial lung disease. Cancer Chemother Pharmacol 74: 1159-1166, 2014.

16. Enomoto Y, Kenmotsu H, Watanabe N, et al. Efficacy and safety of combined carboplatin, paclitaxel, and bevacizumab for patients with advanced non-squamous non-small cell lung cancer with preexisting interstitial lung disease: a retrospective multi-institutional study. Anticancer Res 35: 4259-4263, 2015.

17. American Thoracic Society/European Respiratory Society International Multidisciplinary Consensus Classification of the Idiopathic Interstitial Pneumonias. This joint statement of the American Thoracic Society (ATS), and the European Respiratory Society (ERS) was adopted by the ATS board of directors, June 2001 and by the ERS Executive Committee, June 2001. Am J Respir Crit Care Med 165: 277-304, 2002.

18. Collard HR, Moore BB, Flaherty KR, et al. Acute exacerbations of idiopathic pulmonary fibrosis. Am J Respir Crit Care Med 176: 636-643, 2007.

19. Agarwal R, Jindal SK. Acute exacerbation of idiopathic pulmonary fibrosis: a systematic review. Eur J Intern Med 19: 227-235, 2008.

20. Socinski MA, Langer CJ, Okamoto I, et al. Safety and efficacy of weekly nab(R)-paclitaxel in combination with carboplatin as firstline therapy in elderly patients with advanced non-small-cell lung cancer. Ann Oncol 24: 314-321, 2013.

21. Miyauchi E, Inoue A, Usui K, et al. Phase II study of modified carboplatin plus weekly nab-paclitaxel in elderly patients with non-small cell lung cancer: north Japan lung cancer study group trial 1301. Oncologist 22: e640-e659, 2017.

22. Shiroyama T, Tamiya M, Minami S, et al. Carboplatin plus weekly nanoparticle albumin-bound paclitaxel in elderly patients with previously untreated advanced squamous non-small-cell lung cancer selected based on Mini Nutritional Assessment short-form scores: a multicenter phase 2 study. Cancer Chemother Pharmacol 32: 1103-1106, 2012.

23. Tamiya A, Naito T, Miura S, et al. Interstitial lung disease associated with docetaxel in patients with advanced non-small cell lung cancer. Anticancer Res 32: 1103-1106, 2012.

24. Watanabe N, Niho S, Kirita K, et al. Second-line docetaxel for patients with platinum-refractory advanced non-small cell lung can- cer and interstitial pneumonia. Cancer Chemother Pharmacol 76: 69-74, 2015.

25. Okuda K, Hirose T, Oki Y, et al. Evaluation of the safety and efficacy of combination chemotherapy with vinorelbine and platinum agents for patients with non-small cell lung cancer with interstitial lung disease. Anticancer Res 32: 5475-5480, 2012.

26. Di Maio M, Chiodini P, Georgoulias V, et al. Meta-analysis of single-agent chemotherapy compared with combination chemotherapy as second-line treatment of advanced non-small-cell lung cancer. J Clin Oncol 27: 1836-1843, 2009.

27. Yasuda Y, Hattori Y, Tohnai R, et al. The safety and efficacy of carboplatin plus nanoparticle albumin-bound paclitaxel in the treatment of non-small cell lung cancer patients with interstitial lung disease. Jpn J Clin Oncol 1-5, 2017.

28. Niwa H, Nakahara Y, Yokoba M, Mitsufuji H, Sasaki J, Masuda N. Safety and efficacy of carboplatin plus nab-paclitaxel for treating advanced non-small-cell lung cancer with interstitial lung disease. Mol Clin Oncol 7: 604-608, 2017.

29. Shukuya $T$, Ishiwata $T$, Hara $M$, et al. Carboplatin plus weekly paclitaxel treatment in non-small cell lung cancer patients with interstitial lung disease. Anticancer Res 30: 4357-4361, 2010.

30. Brahmer J, Reckamp KL, Baas $P$, et al. Nivolumab versus docetaxel in advanced squamous-cell non-small-cell lung cancer. $\mathrm{N}$ Engl J Med 373: 123-135, 2015.

31. Reck M, Rodriguez-Abreu D, Robinson AG, et al. Pembrolizumab versus chemotherapy for PD-L1-positive non-small-cell lung cancer. N Engl J Med 375: 1823-1833, 2016.

32. Makimoto $T$, Tsuchiya $S$, Hayakawa $K$, Saitoh R, Mori M. Risk factors for severe radiation pneumonitis in lung cancer. Jpn J Clin Oncol 29: 192-197, 1999.

33. Ohe Y, Yamamoto S, Suzuki K, et al. Risk factors of treatmentrelated death in chemotherapy and thoracic radiotherapy for lung cancer. Eur J Cancer 37: 54-63, 2001.

34. Tsujino K, Hashimoto T, Shimada T, et al. Combined analysis of V20, VS5, pulmonary fibrosis score on baseline computed tomography, and patient age improves prediction of severe radiation pneumonitis after concurrent chemoradiotherapy for locally advanced non-small-cell lung cancer. J Thorac Oncol 9: 983-990, 2014.

35. Ueki N, Matsuo Y, Togashi Y, et al. Impact of pretreatment interstitial lung disease on radiation pneumonitis and survival after stereotactic body radiation therapy for lung cancer. J Thorac Oncol 10: 116-125, 2015.

36. Sanuki N, Ono A, Komatsu E, et al. Association of computed tomography-detected pulmonary interstitial changes with severe radiation pneumonitis for patients treated with thoracic radiotherapy. J Radiat Res 53: 110-116, 2012.

The Internal Medicine is an Open Access article distributed under the Creative Commons Attribution-NonCommercial-NoDerivatives 4.0 International License. To view the details of this license, please visit (https://creativecommons.org/licenses/ by-nc-nd/4.0/).

\section{(C) 2018 The Japanese Society of Internal Medicine} Intern Med 57: 1827-1832, 2018 